\title{
PARACOMPACTNESS AND ELASTIC SPACES
}

\author{
HISAHIRO TAMANO ${ }^{1}$ AND J. E. VAUGHAN
}

ABSTRACT. This paper gives a characterization of paracompactness, and introduces the notion of an elastic space which generalizes the concept of a stratifiable (in particular, metric) space.

1. Introduction. In this note we shall give a characterization of paracompactness which is formally weaker than our previous characterizations [4, Theorem 2], [5, Theorem 3], [6, Theorem 1] concerning linearly cushioned refinements. Furthermore, we shall define a new generalization of metric spaces and stratifiable spaces, called "elastic spaces," by introducing the notion of an "elastic base."

Definition 1. Let $u$ be a collection of subsets of a set $X$, and let $\Re$ be a relation on $u$ (i.e., $\Re \subset \mathcal{u} \times \mathfrak{u}$ ). We shall of ten write $U \Re V$ instead of $(U, V) \in R$. The relation $R$ is said to be a framed relation on $\mathfrak{u}$ (or a framing of $\mathcal{u}$ ) provided for every $U, V \in \mathcal{u}$, if $U \cap V \neq \varnothing$, then $U \Re V$ or $V R U$. We say $R$ is a well-framed relation on $\mathcal{U}$ provided $R$ is a framing of $u$ and for every $x \in X$, there exists an $R$-smallest $U_{x} \in \mathcal{U}$ containing $x$ (i.e., if $x \in U, U \in \mathcal{U}$, and $U \neq U_{x}$, then $\left(U, U_{x}\right) \notin R$ ).

Definition 2. A collection $\mathcal{U}$ is said to be framed in a collection $\mathcal{V}$ with frame map $f: \mathcal{U} \rightarrow \mathcal{U}$. provided there exists a framed relation $R$ on $\mathcal{U}$ such that for every subcollection $\mathcal{U}^{\prime} \subset \mathcal{U}$ which has an $\mathfrak{R}$-upper bound (i.e., there exists $U \in \mathcal{u}$ so that $U^{\prime} \Re U$ for every $U^{\prime} \in \mathcal{U}^{\prime}$ ) we have $\operatorname{cl}\left(\cup \mathcal{u}^{\prime}\right) \subset \cup f\left(\mathcal{u}^{\prime}\right)$. If in addition $R$ is a well-framed relation on $u$, we say that $u$ is well-framed in $v$. Finally, if $u$ is framed in $v$ and $R$ is also a transitive relation, then $\mathcal{U}$ is called elastic in $\mathcal{V}$, or an elastic refinement of $v$ when $U$ and $v$ are covers of $X$.

Theorem 1. Let $X$ be a regular space. A necessary and sufficient condition that $X$ be paracompact is that every open cover of $X$ have an open elastic refinement.

2. Proof of Theorem 1. The proof follows from the next two lemmas.

Presented to the Society, August 28, 1970; received by the editors September 22, 1969.

AMS 1969 subject classifications. Primary 5450, 5435.

Key words and phrases. Paracompact, framing, elastic refinement, elastic base, elastic space, $M_{3}$-space, stratifiable space, metric space.

1 This note is a revision of a manuscript of $\mathrm{H}$. Tamano which was found by J. Nagata after the death of Professor Tamano.

Copyright (c) 1971, American Mathematical Society 
Lemma 1. Let $X$ be a regular space. $A$ necessary and sufficient condition for $X$ to be paracompact is for every open cover of $X$ to have an open refinement which is well-framed in it.

Proof. We shall prove the sufficiency. The proof is similar to that of Theorem 1 in [6]. Let $\mathcal{V}$ be an open cover of $X$. Let $\mathcal{u}$ be an open refinement of $V$ which is well-framed in $V$ with respect to the wellframed relation $R$ on $\mathcal{u}$ and frame map $f: \mathcal{u} \rightarrow \mathcal{V}$. Let $H_{U}=U-$ $U\left\{U^{\prime} \in \mathcal{U}: U^{\prime} R U\right.$ and $\left.U^{\prime} \neq U\right\}$, and $\mathcal{H}=\left\{H_{U}: U \in \mathcal{U}\right\}$. We now show that $\mathfrak{H C}$ is a cushioned refinement of $\mathcal{V}$ with cushion map $g: \mathfrak{H} \rightarrow \mathcal{U}$ defined by $g\left(H_{U}\right)=f(U)$ (these terms are defined in [6]) and conclude that $X$ is paracompact by $[3$, Theorem $1.1, \mathrm{p} .309]$. It is easy to see that $\mathfrak{H}$ is a cover of $X$ since $R$ is well-framed. It remains to show that $\mathfrak{H}$ is cushioned in $\mathcal{V}$. Let $\mathcal{F}^{\prime} \subset \mathcal{F}$, and suppose $x \notin \cup g\left(\mathcal{H}^{\prime}\right)$. Let $U_{x}$ be an $R$-smallest element of $\mathcal{U}$ containing $x$. Clearly $U_{x}$ is an open neighborhood of $x$ missing $H_{U}$ for all $U \neq U_{x}$ such that $U_{x} R U$. Further, $\mathcal{u}^{\prime}=\left\{U \in \mathcal{U}: U \Re U_{x}\right.$ and $\left.H_{U} \in \mathcal{F}^{\prime}\right\}$ has an $\Re$-upper bound. Hence $\operatorname{cl}\left(\cup \mathcal{u}^{\prime}\right) \subset U f\left(\mathcal{U}^{\prime}\right) \subset U_{g}\left(\mathcal{F}^{\prime}\right)$ because $\mathcal{u}$ is framed in $v$. Therefore, there exists an open neighborhood $N$ of $x$ missing $\operatorname{cl}\left(U \mathcal{u}^{\prime}\right)$. Finally, if $U$ is not $R$-related to $U_{x}$, then $U \cap U_{x}=\varnothing$ since $R$ is a framing of $\mathcal{u}$. Thus, $U_{x} \cap N$ is an open neighborhood of $x$ missing $U_{\mathcal{C}^{\prime}}$, and we have $\operatorname{cl}\left(\bigcup_{\mathcal{F}^{\prime}}\right) \subset \bigcup g\left(\mathfrak{H C}^{\prime}\right)$. This completes the proof.

LEMma 2. Let $\mathcal{u}$ be a cover of a set $X$, and let $\leqq$ be a transitive relation which is a framing of $\mathcal{u}$. Then there exists a well-framed relation $R$ on $\mathcal{u}$ so that every subset of $\mathcal{u}$ with an $\mathfrak{R}-$ upper bound has $a \leqq-u p p e r$ bound.

Proof. ${ }^{2}$ Let $\leqq *$ be a well-order on an index set for $\mathcal{U}$ such that $\mathcal{u}=\left\{U_{0}, U_{1}, \cdots, U_{\alpha}, \cdots: \alpha<^{*} \eta\right\}$. Let $\Lambda[\alpha]=\left\{U \in \mathcal{L}: U \leqq U_{\alpha}\right\}$ for all $\alpha<^{*} \eta$. Well-order $\Lambda$ [0] in any manner and denote the well-order by $\leqq \leqq 0$. Suppose a reflexive and antisymmetric relation $\leqq \varliminf_{\beta}$ has been defined on $U\{\Lambda[\gamma]: \gamma \leqq * \beta\}$ for all $\beta<^{*} \alpha$ in such a way that $\leqq \leqq{ }_{\beta}$ is an extension of $\leqq \leqq \delta$ whenever $\delta \leqq * \beta$. Put $\Lambda^{\prime}[\alpha]$ $=\Lambda[\alpha]-\bigcup\left\{\Lambda[\beta]: \beta<^{*} \alpha\right\}$; well-order $\Lambda^{\prime}[\alpha]$ and denote the order by $\leqq_{\alpha}$. Also define $U \leqq_{\alpha} U^{\prime}$ if $U^{\prime} \in \Lambda^{\prime}[\alpha]$ and $U \in \Lambda[\alpha] \cap\left(U\left\{\Lambda[\beta]: \beta<^{*} \alpha\right\}\right)$. Let $\leqq \leqq \alpha$ be the relation on $U\left\{\Lambda[\beta]: \beta \leqq{ }^{*} \alpha\right\}$ generated by $\left\{\leqq \leqq \varliminf_{\beta}: \beta<^{*} \alpha\right\}$ and $\leqq_{\alpha}$. Finally, let $R$ be the reflexive and antisymmetric relation generated by $\left\{\leqq \leqq \alpha: \alpha<{ }^{*} \eta\right\}$.

First we shall show that $\mathcal{R}$ is a framing of $\mathcal{u}$. If $U, U^{\prime} \in \mathcal{U}$ and $U \cap U^{\prime} \neq \varnothing$, then either $U \leqq U^{\prime}$ or $U^{\prime} \leqq U$ since $\leqq$ is a framing of $\mathcal{u}$.

2 The authors would like to thank E. Michael for some helpful suggestions concerning this result. 
Suppose that $U \leqq U^{\prime}$. Let $\alpha_{0}$ be the first index such that $U^{\prime} \in \Lambda\left[\alpha_{0}\right]$. Since $\leqq$ is transitive, we have $U \in \Lambda\left[\alpha_{0}\right]$, and thus by the definition of $a$ we know $U^{\prime} R U$ or $U R U^{\prime}$.

Next we show that if $\mathcal{U}$ has an $R$-upper bound, then $\mathcal{u}$ has a $\leqq$-upper bound. To do this we first note that if $U R U^{\prime}$, then for the first index $\alpha$ such that $U, U^{\prime} \in U\left\{\Lambda[\beta]: \beta \leqq{ }^{*} \alpha\right\}$ we have $U \leqq \leqq_{\alpha} U^{\prime}$, $U^{\prime} \in \Lambda^{\prime}[\alpha]$, and $U \in \Lambda[\alpha]$. To see this, let $\gamma$ be the first index such that $U \leqq \varliminf_{\gamma} U^{\prime}$. Since $\leqq \varliminf_{\gamma}$ is a relation on $U\left\{\Lambda[\beta]: \beta \leqq{ }^{*} \gamma\right\}$ we know $\alpha \varliminf^{*} \gamma$. If $\alpha<^{*} \gamma$, then $U, U^{\prime} \notin \Lambda^{\prime}[\gamma]$ so $U, U^{\prime}$ are not related by $\leqq_{\gamma}$. By the definition of $\leqq \leqq{ }_{\gamma}$, we must have $U \leqq \varliminf_{\beta} U^{\prime}$ for some $\beta<^{*} \gamma$, but this contradicts the definition of $\gamma$. Thus, $\alpha=\gamma$, and $U \leqq \leqq_{\alpha} U^{\prime}$. Further, not both of $U$ and $U^{\prime}$ are in $U\left\{\Lambda[\beta]: \beta<{ }^{*} \delta\right\}$ for any $\delta<^{*} \alpha$, so $U$ and $U^{\prime}$ are not related by $\leqq \leqq_{\delta}$ for any $\delta<^{*} \alpha$. Thus, $U \leqq_{\alpha} U^{\prime}$, from which it follows that $U^{\prime} \in \Lambda^{\prime}[\alpha]$ and $U \in \Lambda[\alpha]$. Now suppose $\mathcal{u}^{\prime}$ is a subcollection of $\mathcal{U}$ which has an $\mathcal{R}$-upper bound. Let $U^{\prime}$ be an R-upper bound of $\mathcal{u}^{\prime}$. Let $\alpha_{0}$ be the first index such that $U^{\prime} \in \Lambda\left[\alpha_{0}\right]$. We now show that $u^{\prime}$ has $U_{\alpha_{0}}$ for $\leqq$-upper bound. Let $U \in \mathcal{u}^{\prime}$ and let $\alpha_{1}$ be the first index such that $U \in \Lambda\left[\alpha_{1}\right]$. If $\alpha_{1} \leqq{ }^{*} \alpha_{0}$, then $\alpha_{0}$ is the first index such that $U, U^{\prime} \in U\left\{\Lambda[\beta]: \beta \leqq * \alpha_{0}\right\}$. Hence $U \Re U^{\prime}$ implies $U, U^{\prime} \in \Lambda\left[\alpha_{0}\right]$ as noted above. In particular $U \leqq U_{\alpha_{0}}$ by definition of $\Lambda\left[\alpha_{0}\right]$. If $\alpha_{0}<^{*} \alpha_{1}$ then $\alpha_{1}$ is the first index such that $U, U^{\prime}$ $\in \bigcup\left\{\Lambda[\beta]: \beta \leqq{ }^{*} \alpha_{1}\right\}$. Hence $U R U^{\prime}$ implies $U^{\prime} \in \Lambda^{\prime}\left[\alpha_{1}\right]$, but this contradicts the fact that $U^{\prime} \in \Lambda\left[\alpha_{0}\right]$.

Finally we show that every nonempty subset $\mathcal{u}^{\prime}$ of $u$ has an R-smallest element. Let $\alpha$ be the first index such that $\mathcal{U}^{\prime} \cap \Lambda[\alpha] \neq \varnothing$. Then $\mathcal{u}^{\prime} \cap \Lambda[\alpha] \subset \Lambda^{\prime}[\alpha]$. Since $\left(\Lambda^{\prime}[\alpha], \leqq_{\alpha}\right)$ is a well-ordered subset of $\mathcal{u}$, there exists a $\leqq{ }_{\alpha}$-first element of $\mathcal{u}^{\prime} \cap \Lambda[\alpha]$ which is an $R$-smallest element of $\mathcal{U}^{\prime}$. Thus $R$ is well-framed, and this completes the proof.

Proof of Theorem 1 . We need only prove the sufficiency. Let $\mathcal{u}$ be an open cover of $X$, and let $w$ be an open elastic refinement of $\mathcal{u}$. By Lemma 2, it is easy to see that there is a well-framed relation on $w$, and that $w$ is well-framed in $\mathcal{U}$. Hence $X$ is paracompact by Lemma 1.

3. Elastic spaces. According to J. G. Ceder [2], a collection $\boldsymbol{P}$ of ordered pairs $P=\left(P_{1}, P_{2}\right)$ of subsets of a space $X$ is called a pair base for $X$ provided that $P_{1}$ is open for all $P \in P$ and that for every $x \in X$ and for every open set $U$ containing $x$, there exists a $P \in P$ such that $x \in P_{1} \subset P_{2} \subset U$. Further, he called a $T_{1}$-space an $M_{3}$-space (renamed stratifiable space by C. J. R. Borges [1]) provided it has a $\sigma$-cushioned pair base $P$. A pair base $\boldsymbol{P}$ is said to be $\sigma$-cushioned provided $\boldsymbol{P}$ 
$=\cup_{n=1}^{\infty} P_{n}$, and for every $n$ and every $P_{n}^{\prime} \subset P_{n}$ we have

$$
\operatorname{cl}\left(\cup\left\{P_{1}: P \in P_{n}^{\prime}\right\}\right) \subset \cup\left\{P_{2}: P \in P_{n}^{\prime}\right\} \text {. }
$$

Definition 3. A pair base $P$ for a space $X$ is said to be an elastic base if there is a framing of $P_{1}=\left\{P_{1}: P=\left(P_{1}, P_{2}\right) \in P\right\}$ such that $P_{1}$ is elastic in $P_{2}=\left\{P_{2}: P=\left(P_{1}, P_{2}\right) \in P\right\}$ with respect to the map $f\left(P_{1}\right)=P_{2}$. A $T_{1}$-space with an elastic base is called an elastic space.

THEOREM 2. Every subspace of an elastic space is an elastic space. Every metrizable space, and more generally every stratifiable space, is an elastic space. Every elastic space is paracompact.

Proof. The first statement is obvious. Let $X$ be a stratifiable space with a $\sigma$-cushioned pair base $P=\cup_{n=1}^{\infty} P_{n}$. We may assume that $\left\{P_{n}: n=1,2, \cdots\right\}$ is a partition of $P$. Let $\leqq_{n}$ be a well-order on $P_{n}$ for each $n$, and define a well-order $\leqq$ on $P$ as follows: For $P, P^{\prime} \in P$ we say $P \leqq P^{\prime}$ if and only if either (1) $P, P^{\prime}$ are in the same $P_{n}$ and $P \leqq{ }_{n} P^{\prime}$, or (2) $P \in P_{n}, P^{\prime} \in P_{m}$, and $n<m$. Then $P$ obviously is an elastic base. Since an elastic space is regular, it follows from Theorem 1 that every elastic space is paracompact.

ExAmple. (An elastic space which is not a stratifiable space.) Let $X=[0, \Omega]$ be the set of ordinals less than or equal to the first uncountable ordinal. Let the topology on $X$ be the weakest topology stronger than the order topology for which every point is isolated except $\Omega$. Construct an elastic base for $X$ as follows. Let $U_{\alpha}=(\alpha, \Omega]$ for all $\alpha<\Omega$, and let $P^{\prime}=\left\{\left(U_{\alpha}, U_{\alpha}\right): \alpha \in[0, \Omega)\right\}$ and order $P^{\prime}$ by the usual order on the index set $[0, \Omega)$. Let $W_{\alpha}=\{\alpha\}$ for all $\alpha<\Omega$, and let $P^{\prime \prime}=\left\{\left(W_{\alpha}, W_{\alpha}\right): \alpha \in[0, \Omega)\right\}$ and order $P^{\prime \prime}$ by the usual order on the index set $[0, \Omega)$. Finally, set $P=P^{\prime} \cup P^{\prime \prime}$ and order $P$ so that every element of $P^{\prime}$ precedes every element of $P^{\prime \prime}$. Then $P$ is an elastic base for $X$, so $X$ is an elastic space. Clearly, $X$ is not stratifiable because the closed set $\{\Omega\}$ is not a $G_{\delta}$ in $X$ (see [2, Theorem 2.2, p. 106]).

CONJECTURE. ${ }^{3}$ Every closed continuous image of an elastic space is an elastic space.

\section{REFERENCES}

1. C. J. R. Borges, On stratifiable spaces, Pacific J. Math. 17 (1966), 1-16. MR $32 \# 6409$.

2. J. G. Ceder, Some generalizations of metric spaces, Pacific J. Math. 11 (1961), 105-125. MR 24 \#A1707.

3 Tamano called this statement a theorem in his manuscript, but he did not give a complete proof; so it is stated here as a conjecture. 
3. E. Michael, Yet another note on paracompact spaces, Proc. Amer. Math. Soc. 10 (1959), 309-314. MR 21 \#4406.

4. H. Tamano, $A$ characterization of paracompactness, Fund. Math. (to appear). 5. ——, On some characterizations of paracompactness, Topology Conference (Arizona State University, Tempe, Arizona, 1967), Arizona State Univ., Tempe, Ariz., 1968, pp. 277-285. MR 38 \#6539.

6. J. E. Vaughan, Linearly ordered collections and paracompactness, Proc. Amer. Math. Soc. 24 (1970), 186-192. MR 40 \#6503.

Texas Christian University, Fort Worth, Texas 76129

University of North Carolina, Chapel Hill, North Carolina 27514 